\title{
168-Gb/s All-Optical Wavelength Conversion With a Symmetric-Mach-Zehnder-Type Switch
}

\author{
Shigeru Nakamura, Yoshiyasu Ueno, and Kazuhito Tajima
}

\begin{abstract}
Error-free all-optical wavelength conversion at $168 \mathrm{~Gb} / \mathrm{s}$, which is the highest repetition rate ever reported, has been achieved by using a Symmetric-Mach-Zehnder (SMZ)-type switch. Low-power-penalty 84-Gb/s operation is also demonstrated. The push-pull switching mechanism of the SMZ switch enables such ultrafast operation based on cross-phase modulation associated with the carrier depletion in a semiconductor optical amplifier. The configuration of the Delayed-Interference Signal-wavelength Converter, which is a simplified variant of the SMZ switch, is used in this experiment.
\end{abstract}

Index Terms-All-optical switch, interferometer, nonlinear optics, semiconductor optical amplifier, ultrafast, wavelength conversion.

\section{INTRODUCTION}

$\mathbf{T}$ HE SCHEME of ultrafast optical networks based on optical-time-division multiplexing (OTDM) technology attracts much attention in terms of not only high capacity but also high flexibility. In such networks, the expected bit rates per wavelength channel are over $100 \mathrm{~Gb} / \mathrm{s}$, and thus various signal processing at these ultrahigh bit rates will be done in the optical domain. In achieving such ultrafast optical signal processing, the Symmetric-Mach-Zehnder (SMZ) all-optical switch family, including the original SMZ switch [1], the Polarization-Discriminating SMZ (PD-SMZ) switch [2], and the Delayed-Interference Signal-wavelength Converter (DISC) [3]-[5] is quite promising. These all-optical switches use essentially the same mechanism to enable high-speed and high-efficiency switching. Although switching is based on a refractive index change induced by a carrier density change in a semiconductor, the push-pull switching mechanism cancels out the effect associated with slow relaxation of the induced refractive index change. The applicability of the SMZ switch family to ultrafast demultiplexing (DEMUX) has already been verified in various experiments. We have demonstrated 200 -fs switching [6], showing the capability of over $1-\mathrm{Tb} / \mathrm{s}$ DEMUX. Recently, error-free $168-\mathrm{Gb} / \mathrm{s}$ DEMUX with the hybrid-integrated SMZ (HI-SMZ) switch [7] has also been demonstrated. However, to utilize the SMZ switches for a wider range of applications such as logic, regeneration [8], or wavelength conversion [9], operation excited by higher repetition data-modulated optical pulses is required on top of

Manuscript received January 18, 2001; revised June 18, 2001. This work was supported in part by the Femtosecond Technology Project under the management of the Femtosecond Technology Research Association supported by the New Energy and Industrial Technology Development Organization.

The authors are with System Devices and Fundamental Research, NEC Corporation, 305-8501 Ibaraki, Japan (e-mail: s-nakamura@dy.jp.nec.com).

Publisher Item Identifier S 1041-1135(01)08074-0. being ultrafast. To date, $100-\mathrm{Gb} / \mathrm{s}$ wavelength conversion [10] and $80-\mathrm{Gb} / \mathrm{s}$ pulse regeneration [11] have been shown by using the push-pull switching mechanism of the SMZ all-optical switch incorporating semiconductor optical amplifiers (SOAs). In such operation, the pattern effect due to high repetition data-modulated signal pulses is suppressed by injecting unmodulated continuous-wave (CW) light or clock pulses into SOAs at relatively high average power [4], [12]. Several experiments [10] exhibiting ultrafast all-optical wavelength conversion accompanied the conversion from return-to-zero (RZ) to nonreturn-to-zero (NRZ) and/or logic inversion. Although such format conversion is also useful depending on applications, unchanging pulse duration and noninverting logic would be generally required.

Here, we report on the first error-free $168-\mathrm{Gb} / \mathrm{s}$ all-optical wavelength conversion by using the DISC. Low-power-penalty $84-\mathrm{Gb} / \mathrm{s}$ operation is also demonstrated. The results confirm that the SMZ-type switches can be driven by data-modulated signal pulses at a bit rate of $160 \mathrm{~Gb} / \mathrm{s}$, which is considered to be the bit rate for the first-generation OTDM system. The wavelength converter operates without logic inversion and keeps the pulse duration almost unchanged from input to output.

\section{EXPERIMENTAL SETUP}

The experimental setup for $168-\mathrm{Gb} / \mathrm{s}$ wavelength conversion is shown schematically in Fig. 1. The DISC consists of a nonlinear waveguide and a delay line, as detailed in [3]. An SOA module was used as the nonlinear waveguide. In the SOA, signal pulses deplete carriers, and thus give cross-phase modulation to CW light at another wavelength. At the delay line, the two temporally displaced components of the phase-modulated CW light interfere, forming a pulse output. In this experiment, the delay line was composed of a calcite, a Babinet-Soleil phase shifter, and a polarizer. The duration of the output pulse from the DISC was determined by the birefringence of the calcite and was unrestricted by the slow relaxation of the induced nonlinear phase shift.

The optical source for the signal pulses was an actively modelocked fiber laser (PriTel, Inc.) that generated 1.0-ps 1564-nm pulses at a repetition rate of $10.5 \mathrm{GHz}$. The $168 \mathrm{-Gb} / \mathrm{s}$ signal pulses were generated by modulating the output of the fiber laser with a pseudorandom bit sequence (PRBS) with a length of $2^{7}-1$ and then passively multiplexing the modulated pulses. To obtain uniformly multiplexed signal pulses, we used twostage fiber-based delay lines and two-stage spatial delay lines, where the intensities and intervals of multiplexed pulses were adjustable. These $168-\mathrm{Gb} / \mathrm{s}$ signal pulses were injected into the 


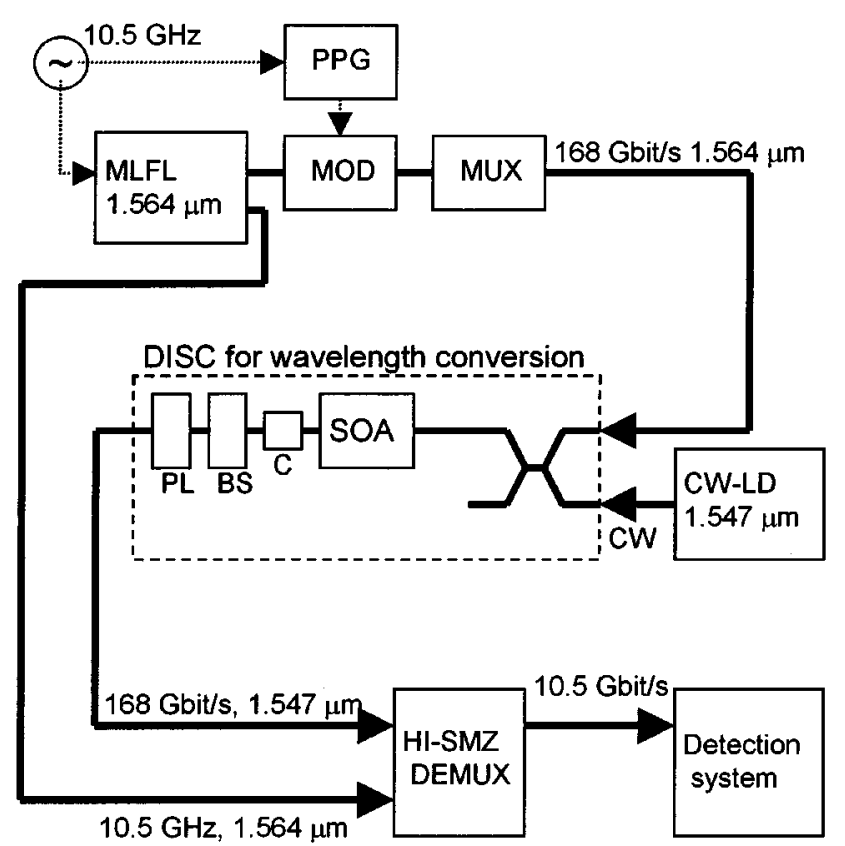

Fig. 1. Experimental setup. MLFL: Mode-locked fiber laser. MOD: Modulator. PPG: Pulse pattern generator. C: Calcite. BS: Babinet-Soleil phase shifter. PL: Polarizer.

DISC together with unmodulated 1547-nm CW light generated by an external-cavity semiconductor laser. The injection current for the SOA was set to $250 \mathrm{~mA}$. The average powers of the signal pulses and of the $\mathrm{CW}$ light at the input of the SOA module were 10 and $16 \mathrm{dBm}$, respectively. The signal pulse energy coupled to the SOA was estimated to be $240 \mathrm{fJ}$. The signal pulse duration at the input of the DISC was $1.8 \mathrm{ps}$. The time delay provided by the birefringence of the calcite was set to $2.0 \mathrm{ps}$, which determined the duration of the output pulse. Thus, the pulse duration was kept almost unchanged before and after wavelength conversion. Also note that logic was not inverted in this wavelength conversion. The $168-\mathrm{Gb} / \mathrm{s}$ output of the DISC was demultiplexed to $10.5 \mathrm{~Gb} / \mathrm{s}$ by the HI-SMZ switch [7] to measure eye diagrams and bit error rates (BERs).

\section{RESUlTS AND DisCUSSION}

Fig. 2(a) shows the 168-Gb/s output waveform of the DISC measured by a streak camera. The trace was recorded as the accumulation of many PRBS pulses, and thus was observed as a regular pulse sequence. The trace indicates good uniformity for all 16 multiplexed channels. From a trace measured with a higher time resolution ( $1.3 \mathrm{ps})$, shown in the inset of Fig. 2(a), the extinction ratio was estimated to be more than $10 \mathrm{~dB}$. An output pulse duration of $1.9 \mathrm{ps}$ was confirmed by the autocorrelation measurement as shown in Fig. 2(b).

Fig. 3 is the eye diagram for the $168-\mathrm{Gb} / \mathrm{s}$ output of the DISC measured after DEMUX. A clear eye opening has been achieved. Fig. 4(a) is the result of BER measurement, showing error-free $168-\mathrm{Gb} / \mathrm{s}$ wavelength conversion. All 16 multiplexed channels showed similar BER performance. For comparison, a BER measurement result for error-free $84-\mathrm{Gb} / \mathrm{s}$ operation is also shown in Fig. 4(b). (In 84-Gb/s operation, a pattern length of $2^{31}-1$ was used to drive the $10.5-\mathrm{Gb} / \mathrm{s}$ modulator
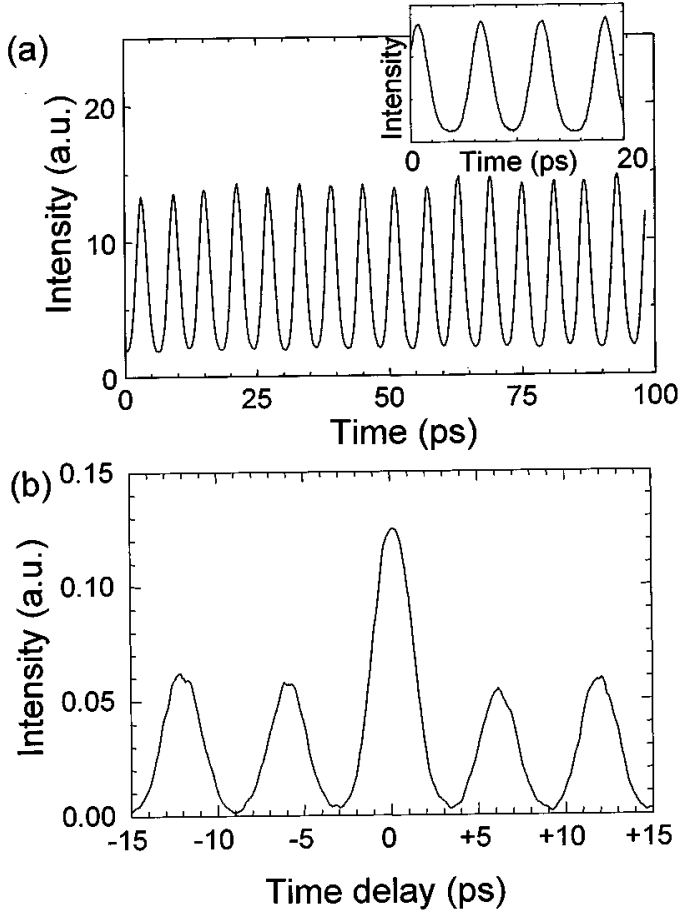

Fig. 2. (a) Streak camera trace for DISC output. Inset is a trace measured at a higher time resolution. (b) Auto-correlater trace for DISC output. The full-width at half-maximum of the trace is $2.9 \mathrm{ps}$, indicating a duration of $1.9 \mathrm{ps}$ when the $\operatorname{sech}^{2}$ pulse shape is assumed.

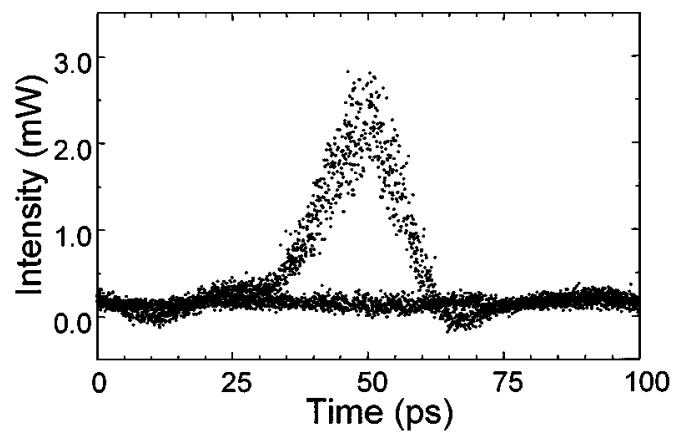

Fig. 3. Eye diagram for DISC output after DEMUX.
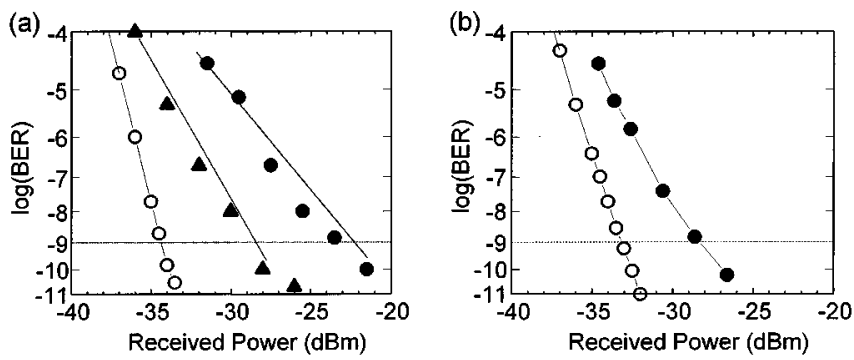

Fig. 4. (a) BER measurement results for $168 \mathrm{~Gb} / \mathrm{s}$. $\bigcirc 10.5-\mathrm{Gb} / \mathrm{s}$ baseline. $\mathbf{A}$ MUX + DEMUX at $168 \mathrm{~Gb} / \mathrm{s}$. $\bullet$ MUX + wavelength conversion + DEMUX at $168 \mathrm{~Gb} / \mathrm{s}$. (b) BER measurement results for $84 \mathrm{~Gb} / \mathrm{s}$. $\bigcirc 10.5-\mathrm{Gb} / \mathrm{s}$ baseline. - MUX + wavelength conversion + DEMUX at $84 \mathrm{~Gb} / \mathrm{s}$.

before MUX.) The received power was measured for $10.5-\mathrm{Gb} / \mathrm{s}$ signals fed into the preamplifier erbium doped fiber amplifier (EDFA) of the detection system. The power penalty measured from the $10.5-\mathrm{Gb} / \mathrm{s}$ baseline includes the effects of MUX and DEMUX. For $84-\mathrm{Gb} / \mathrm{s}$ operation, the power penalty for 
MUX and DEMUX was estimated to be about $2.5 \mathrm{~dB}$ from a separate experiment [7]. Thus, the power penalty attributed to 84-Gb/s wavelength conversion was as low as $2 \mathrm{~dB}$ at a BER of $10^{-9}$. The power penalty for $168-\mathrm{Gb} / \mathrm{s}$ wavelength conversion increased to about $6 \mathrm{~dB}$. These power penalties for wavelength conversion are mainly due to a residual pattern effect induced in the SOA by the data-modulated signal pulses. Because of the pattern effect, we did not reach error-free operation with longer PRBS lengths. The mechanism of suppressing the pattern effect by using the relatively high average power of the $\mathrm{CW}$ light is interpreted by the effective reduction in the carrier lifetime of the SOA [4], [12]. For higher repetition, effective carrier lifetime should be decreased by increasing the power of the $\mathrm{CW}$ light. However, the intense $\mathrm{CW}$ light consumes the gain of the SOA, and thus reduces the refractive index change induced by the signal pulses. Thus, minimizing the pattern effect and maximizing the refractive index change should be balanced at a high repetition rate. We believe that this tradeoff is overcome by developing SOAs in which higher current injection is enabled. Although the observed performance depended on the polarization of the input signal pulses at present, the operation can be optimized to be polarization-independent in principle.

\section{CONCLUSION}

We have achieved error-free all-optical wavelength conversion at $168 \mathrm{~Gb} / \mathrm{s}$, which is the highest repetition rate ever reported, with the Delayed Interference Signal-wavelength Converter. Low-power-penalty $84-\mathrm{Gb} / \mathrm{s}$ operation has also been achieved. We believe that the present operation is more complete than earlier $100-\mathrm{Gb} / \mathrm{s}-\mathrm{level}$ experiments because the pulse duration is kept nearly unchanged, logic is noninverted, and high extinction ratio output is obtained. The results confirm that the Symmetric-Mach-Zehnder-type all-optical switches can be driven by data-modulated $160-\mathrm{Gb} / \mathrm{s}$ signal pulses and are applicable to various optical signal processing in future OTDM systems.

\section{REFERENCES}

[1] K. Tajima, "All-optical switch with switch-off time unrestricted by carrier lifetime," Jpn. J. Appl. Phys., pt. 2, vol. 32, no. 12A, pp. L1746-L1749, Dec. 1993.

[2] K. Tajima, S. Nakamura, and Y. Sugimoto, "Ultrafast polarization-discriminating Mach-Zehnder all-optical switch," Appl. Phys. Lett., vol. 67, no. 25, pp. 3709-3711, June 1995.

[3] Y. Ueno, S. Nakamura, K. Tajima, and S. Kitamura, "3.8-THz wavelength conversion of picosecond pulse using a semiconductor delayed-interference signal wavelength converter (DISC)," IEEE Photon. Technol. Lett., vol. 10, pp. 346-348, Oct. 1998.

[4] Y. Ueno, S. Nakamura, and K. Tajima, "Ultrafast 168-GHz 1.5-ps 1-fJ symmetric-Mach-Zehnder-type all-optical semiconductor switch," Jpn. J. Appl. Phys., pt. 2, vol. 39, no. 8A, pp. L806-L808, Aug. 2000.

[5] Y. Ueno, S. Nakamura, H. Hatakeyama, T. Tamanuki, T. Sasaki, and K. Tajima, "168-Gb/s OTDM wavelength conversion using an SMZ-type all-optical switch," in Proc. 26th Eur. Conf. Optical Communication 2000, vol. 1, Munich, Germany, Sept., pp. 13-14.

[6] S. Nakamura, Y. Ueno, and K. Tajima, "Ultrafast (200-fs switching, $1.5-\mathrm{Tb} / \mathrm{s}$ demultiplexing) and high-repetition $(10 \mathrm{GHz})$ operations of a polarization-discriminating symmetric Mach-Zehnder all-optical switch," IEEE Photon. Technol. Lett., vol. 10, pp. 1575-1577, Nov. 1998.

[7] S. Nakamura, Y. Ueno, K. Tajima, J. Sasaki, T. Sugimoto, T. Kato, T. Shimoda, M. Itoh, H. Hatakeyama, T. Tamanuki, and T. Sasaki, "Demultiplexing of $168-\mathrm{Gb} / \mathrm{s}$ data pulses with a hybrid-integrated symmetric Mach-Zehnder all-optical switch," IEEE Photon. Technol. Lett., vol. 12, pp. 425-427, Apr. 2000.

[8] S. Fischer, M. Dülk, E. Gamper, W. Vogt, E. Gini, H. Melchior, W. Hunziker, D. Nesset, and A. D. Ellis, "Optical 3R regenerator for $40-\mathrm{Gb} / \mathrm{s}$ networks," Electron. Lett., vol. 35, no. 23, pp. 2047-2049, Nov. 1999.

[9] B. Mikkelsen, K. S. Jepsen, M. Vaa, H. N. Poulsen, K. E. Stubkjaer, R. Hess, M. Duelk, W. Vogt, E. Gamper, E. Gini, P. A. Besse, H. Melcior, S. Bouchoule, and F. Devaux, "All-optical wavelength converter scheme for high-speed RZ signal formats," Electron. Lett., vol. 33, pp. 2138-2139, Dec. 1997.

[10] J. Leuthold, C. H. Joyner, B. Mikkelsen, G. Raybon, J. L. Pleumeekers, B. I. Miller, K. Dreyer, and C. A. Burrus, "100-Gb/s all-optical wavelength conversion with integrated SOA delayed-interference configuration," Electron. Lett., vol. 36, pp. 1129-1130, June 2000.

[11] A. E. Kelly, I. D. Phillips, R. J. Manning, A. D. Ellis, D. Nesset, D. G. Moodie, and R. Kashyap, "80-Gb/s all-optical regenerative wavelength conversion using semiconductor optical amplifier based interferometer," Electron. Lett., vol. 35, pp. 1477-1478, Aug. 1999.

[12] R. J. Manning, A. D. Ellis, A. J. Poustie, and K. J. Blow, "Semiconductor laser amplifiers for ultrafast all-optical signal processing," J. Opt. Soc. Amer. B, Opt. Phys., vol. 14, pp. 3204-3216, Nov. 1997. 